\title{
Multiple Spin State Analysis in Radical Carbon Edge and Oxygen Edge Graphene-like Molecules
}

\author{
Norio Ota, Narjes Gorjizadeh*, and Yoshiyuki Kawazoe* \\ Pure and Applied Sciences, University of Tsukuba, 1-1-1 Tenoudai, Tsukuba 305-8573 Japan \\ * Institute for Materials Research, Tohoku University, 2-1-1 Katahira, Aoba-ku, Sendai 980-8577 Japan
}

\begin{abstract}
We propose analysis of multiple spin states in radical carbon zigzag edge graphene-like molecules. Calculations based on density function theory (DFT) revealed that the highest spin state was most stable in every radical carbon edge asymmetric molecule. A typical $\mathrm{C}_{64} \mathrm{H}_{17}$ molecule has five unpaired electrons, which creates three possible molecular spin states of $\mathrm{Sz}_{\mathrm{z}}=1 / 2,3 / 2$, and 5/2. The $\mathrm{Sz}=5 / 2$ state was the most stable among these states. Such DFT results coincided with a simple magnetic counting rule to give a localized spin of $\mathrm{Sz}=+2 / 2$ to one radical carbon site, whereas it gave $\mathrm{Sz}=-1 / 2$ at the nearest carbon and $+1 / 2$ at the second nearest. A total molecular spin of $\mathrm{Sz}$ was obtained by using the simple sum of those numbers. Radical carbon zigzag edge nano-graphene is a promising candidate for designing new materials with strong magnetism. In addition, we studied oxygen-substituted zigzag edges occupied by four electrons. These four spins canceled each other out in two tetrahedral orbits, which led to weak molecular magnetism.
\end{abstract}

Keywords: graphene, spin state, radical carbon, oxygen, first principles calculation

\section{Introduction}

Carbon-based metal-free ferromagnetic materials are very attractive from both the scientific aspect of pi-electron oriented magnetism and the technological aspect of lightweight ecological magnets ${ }^{1-4)}$ and novel spintronic devices ${ }^{5)-9)}$.

Several experiments ${ }^{10)}{ }^{15}$ ) have predicted the capability of room-temperature (RT) ferromagnetism in graphite-like materials. Ohldag et al. ${ }^{15)}$ have recently demonstrated that proton ion implanted pure graphite exhibits strong surface magnetism. The saturation magnetization was estimated to be $12-15 \mathrm{emu} / \mathrm{g}$ at 300 $\mathrm{K}$. This value is almost a quarter that of metal nickel magnetization. Saito et al. ${ }^{12)}$ developed ferromagnetic graphite-like carbon material by using a method of pyrolysis, which had a saturation magnetization of 1.22 emu/g at RT. Although these experiments have motivated us to create promising new materials, suitable models and guiding principles on how to design these materials have not yet been clarified. This paper focuses on the analysis of multiple spin states in radical carbon edge and oxygen edge graphene-like molecules as a promising candidate to demonstrate strong magnetism.

Many theoretical predictions have been made on graphene zigzag edge carbon magnetism due to localized states near the Fermi energy ${ }^{16)-20)}$. Fujita et al. ${ }^{16)}$ focused on infinite length graphene ribbons and applied a tight binding model resulting in an antiferromagnetic feature with zero total magnetization. Rectangular graphene has been extensively studied ${ }^{21)-24)}$. The singlet state (zero magnetization) arises through modifications to both zigzag edges with various species such as $-\mathrm{H},-\mathrm{F},-\mathrm{O}$, $-\mathrm{OH}$, and $-\mathrm{CH}_{3}{ }^{23)}$ In contrast, Kusakabe and Maruyama ${ }^{25)}$, 26) proposed an asymmetric infinite length ribbon model showing ferrimagnetic behavior with non-zero total magnetization.
Our previous papers ${ }^{27)}{ }^{28)}$ reported analysis of multiple spin states in asymmetric graphene molecules using first principles density function theory (DFT). In particular, the most stable energy states in a dihydrogenated zigzag edge graphene molecule were the highest spin state $^{28)}$. However, detailed magnetic analysis has not yet been applied to radical carbon edge molecules. This paper discusses our examination of the behavior of multiple spin states in radical carbon edge molecules. In addition, the magnetism of an oxygen substituted zigzag edge molecule is well understood under similar considerations.

\section{Model Molecules}

A proton ion irradiation experiment ${ }^{10)}$ inspired us to build an appropriate model with piled-up graphene, as seen in Fig. 1. Protons are irradiated from the top. The second layer from the top shows that bare zigzag edge carbons are attacked on the right hand side by protons and modified into a mono-hydrogenated carbon site. In contrast, the other side edge hidden by a top upper molecule remains a radical carbon site due to a shadow effect. The molecule model is favorable toward obtaining total molecular energy with a relative accuracy of $10 \mathrm{E}-8$ depending on the respective spin state, and it is easy to compare the spin density map in real space. The model molecule has a neutral total charge, whereas it has various unpaired electrons. For example, $\mathrm{C}_{64} \mathrm{H}_{17}$ has five radical carbon edges as like shown in Fig. 2(a), which gives five unpaired electrons. Five electrons create five molecular orbits, which allow three possible spin states of Sz. The highest allowable spin state is $\mathrm{Sz}=5 / 2$, the next is $3 / 2$, and the lowest is $1 / 2$. Five molecules were used as the typical calculation models illustrated in Fig. 2. To compare the dependence of spin configuration on edge number, the 
numbers of radical carbons were changed like those in (a) $\mathrm{C}_{64} \mathrm{H}_{17}$ with five radical carbons and with capable spin states of $\mathrm{Sz}_{\mathrm{z}}=5 / 2,3 / 2$, and $1 / 2$, (b) $\mathrm{C}_{56} \mathrm{H}_{16}$ with four radical carbons and with capable spin states of $\mathrm{Sz}=4 / 2$, $2 / 2$, and $0 / 2$, (c) $\mathrm{C}_{64} \mathrm{H}_{19}$ with three radical carbons and with capable spin states of $\mathrm{Sz}=3 / 2$ and $1 / 2$, (d) $\mathrm{C}_{56} \mathrm{H}_{18}$ with two radical carbons and with capable spin states of $\mathrm{Sz}=2 / 2$ and $0 / 2$, and (e) $\mathrm{C}_{64} \mathrm{H}_{21}$ with one radical carbon and with a capable spin state of $1 / 2$.

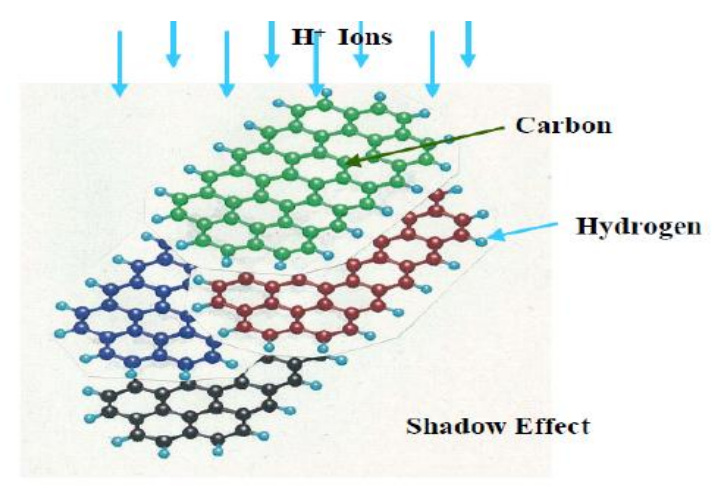

Fig. 1 Graphene-like molecule model: Graphene molecules are piled up one by one, where proton ions are irradiated from the top. It is clear by looking at the second layer from the top that bare zigzag edge carbons on the right hand side are attacked by protons and modified into a mono-hydrogenated carbon site. In contrast, on the other side edge hidden under a top molecule, radical carbon remains due to a shadow effect.
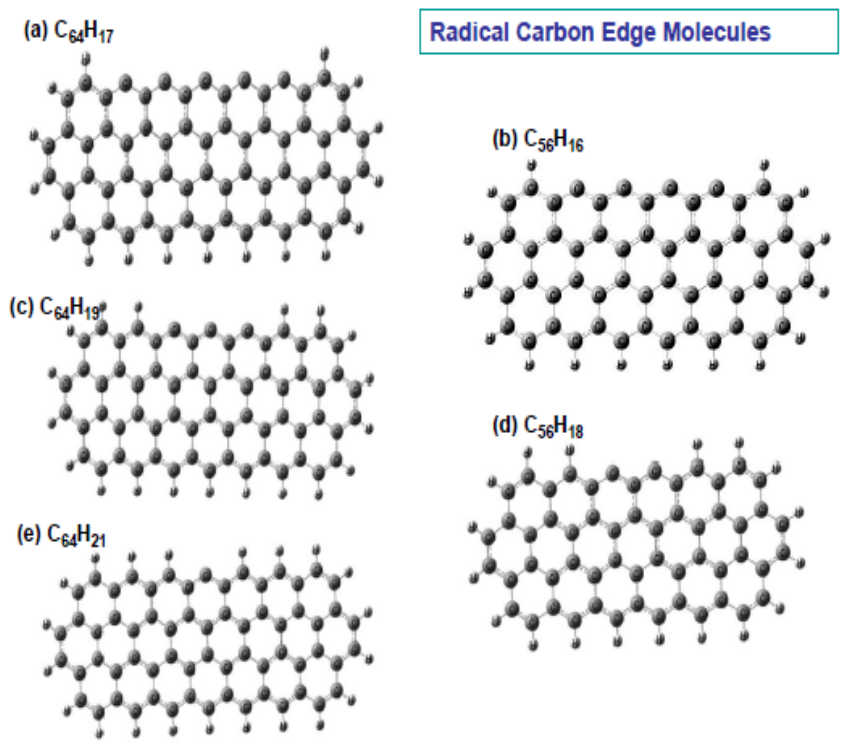

Fig. 2 Model molecules with radical carbon zigzag edges: (a) $\mathrm{C}_{64} \mathrm{H}_{17}$, (b) $\mathrm{C}_{56} \mathrm{H}_{16}$, (c) $\mathrm{C}_{64} \mathrm{H}_{19}$, (d) $\mathrm{C}_{56} \mathrm{H}_{18}$, and (e) $\mathrm{C}_{64} \mathrm{H}_{21}$, where large balls are carbon atoms and small ones are hydrogen.

\section{Methods of Calculation}

To clarify the magnetism, we have to obtain spin density, total molecular energy, and the optimized atom arrangement depending on the respective given molecular spin states of Sz. The DFT calculations were started after the given $\mathrm{Sz}$ was installed. A density function theory (DFT) ${ }^{33)}$, 34) based method of generalized gradient approximation (GGA-UPBEPBE) ${ }^{35)}$ was used. The atomic orbital basis was $6-31 G^{36}$. A relative energy accuracy of at least $10 \mathrm{E}-8$ was required as total molecular energy after the optimization of the atom position was repeated within 128 cycles.

\section{Radical Carbon Edge Molecule}

Every molecule has allowable numbers of unpaired electrons, which enable limited numbers of multiple spin states. For example, there are three spin states like $\mathrm{Sz}_{\mathrm{z}}=1 / 2,3 / 2$, and $5 / 2$ in $\mathrm{C}_{64} \mathrm{H}_{17}$ with five unpaired electrons. These $\mathrm{Sz}$ values are installed as spin parameter "given Sz" to start the DFT calculations. Typical spin density maps are shown in Fig. 3, comparing $\mathrm{Sz}=2 / 2$ and $4 / 2$ in $\mathrm{C}_{56} \mathrm{H}_{16}$ with four unpaired electrons, and $\mathrm{Sz}=1 / 2$ and $3 / 2$ in $\mathrm{C}_{64} \mathrm{H}_{19}$ with three electrons, where up-spins are indicated in red (dark gray), and down-spins in blue (light gray) at the $0.001 \mathrm{e} / \mathrm{A}^{3}$ contour spin density surface. We found that up and down spin clouds were very regularly aligned one by one in every highest spin state $\left(\mathrm{Sz}=4 / 2\right.$ in $\mathrm{C}_{56} \mathrm{H}_{16}$ and $3 / 2$ in $\mathrm{C}_{64} \mathrm{H}_{19}$ ). In contrast, a very complex spin structure appeared inside a molecule like up-up and down-down spin pairs in lower spin states $(\mathrm{Sz}=2 / 2$ in $\mathrm{C}_{56} \mathrm{H}_{16}$ and $1 / 2$ in $\mathrm{C}_{64} \mathrm{H}_{19}$ ). Exchange coupling between up-up spins (also down-down) brought about a local exchange energy increase, and this finally elevated the total molecular energy. Exact DFT-based total molecular energy calculations were done. The results are summarized in Table 1 and Fig. 4 , where $\Delta \mathrm{E}$ is the energy difference from the lowest energy. For example, among the three spin states in $\mathrm{C}_{64} \mathrm{H}_{17}$, the lowest (stable) energy state is the highest spin state of $\mathrm{Sz}=5 / 2$ and the next is $\mathrm{Sz}=3 / 2$. The energy difference, $\Delta \mathrm{E}$, between these two states was $2.5 \mathrm{kcal} / \mathrm{mol}$.

We compared five molecules. The most stable spin state in every molecule was the highest one, i.e., it did not depend on the numbers of radical carbons. The next stable spin state was the next lowest $\mathrm{Sz}$, e.g., in $\mathrm{C}_{64} \mathrm{H}_{17}$ the next lowest was $3 / 2$. The most unstable spin state was the lowest in every molecule. This is because reduced spin states require complex up-up (also down-down) spin pairs inside a molecule to balance with the total numbers of unpaired electrons. We should check the dependence of stable spin state arrangements on edge numbers. Changing the numbers from 5 to 1 , we can see from Fig. 4 that all molecules have a large local up-spin cloud specifically at all radical carbon sites. It should be noted that such edge specified spin configurations govern whole spin arrangements in a molecule.

The DFT square spin density $\mathrm{S}(\mathrm{S}+1)$ value is a 
parameter to check the accuracy of spin configurations as summarized in Table 1. The given $\mathrm{Sz}(\mathrm{Sz}+1)$ is a simple estimation using the given $\mathrm{Sz}$. We can see that DFT $\mathrm{S}(\mathrm{S}+1)$ does not necessarily agree with the given $\mathrm{Sz}(\mathrm{Sz}+1)$. For $\mathrm{Sz}=5 / 2$ of $\mathrm{C}_{64} \mathrm{H}_{17}, \mathrm{~S}(\mathrm{~S}+1)$ is 9.78 , whereas $\mathrm{Sz}(\mathrm{Sz}+1)$ is 8.75 , which is almost a $12 \%$ difference. This means the so called spin-contamination effect depends on the method of calculation. The method of evaluating electron-electron correlation affects spin contamination. We checked the Hartree-Fock and advanced higher order perturbation methods, where the resulting spin contamination unfortunately exceeded 200\%. DFT-GGA approximation is currently a better choice to reduce such spin contamination as much as possible.

Table 1 Results from DFT calculations: The given $\mathrm{Sz}$ is a spin parameter to start DFT calculations, whereas $\mathrm{Sz}$ estimated by using a simple magnetic counting rule is given in the rightmost column. DFT obtained $\mathrm{S}(\mathrm{S}+1)$ is compared with given $\mathrm{Sz}(\mathrm{Sz}+1)$ to check for spin-contamination. $\Delta \mathrm{E}$ is the energy difference from the lowest energy.

\begin{tabular}{|c|c|c|c|c|c|}
\hline Chemical Formula & Given $\mathrm{Sz}$ & $\begin{array}{l}\text { Given } \\
\mathrm{Sz}(\mathrm{Sz}+1)\end{array}$ & $\begin{array}{c}\text { DFT } \\
S(S+1)\end{array}$ & $\begin{array}{l}\text { DFT } \\
\Delta \mathrm{E}(* 1)\end{array}$ & $\begin{array}{l}\text { Sz by a } \\
\text { magnetic } \\
\text { counting rule }\end{array}$ \\
\hline \multirow[t]{3}{*}{$\mathrm{C}_{64} \mathrm{H}_{17}$} & $5 / 2$ & 8.75 & 9.78 & 0.0 & $5 / 2$ \\
\hline & $3 / 2$ & 3.75 & 5.59 & 2.5 & - \\
\hline & $1 / 2$ & 0.75 & 3.20 & 5.8 & - \\
\hline \multirow[t]{3}{*}{$\mathrm{C}_{56} \mathrm{H}_{16}$} & $4 / 2$ & 6.00 & 6.75 & 0.0 & $4 / 2$ \\
\hline & $2 / 2$ & 2.00 & 3.32 & 1.9 & - \\
\hline & $0 / 2$ & 0.00 & $(* 2)$ & $(* 2)$ & - \\
\hline \multirow[t]{2}{*}{$\mathrm{C}_{64} \mathrm{H}_{19}$} & $3 / 2$ & 3.75 & 4.68 & 0.0 & $3 / 2$ \\
\hline & $1 / 2$ & 0.75 & 2.39 & 2.9 & - \\
\hline \multirow[t]{2}{*}{$\mathrm{C}_{56} \mathrm{H}_{18}$} & $2 / 2$ & 2.00 & 3.32 & 0.0 & $2 / 2$ \\
\hline & $0 / 2$ & 0.00 & $(* 2)$ & $(* 2)$ & - \\
\hline $\mathrm{C}_{64} \mathrm{H}_{21}$ & $1 / 2$ & 0.75 & 0.86 & 0.0 & $1 / 2$ \\
\hline
\end{tabular}

(*1) Energy difference from the lowest state $(\mathrm{kcal} / \mathrm{mol})$ (*2) SCF calculation does not converged

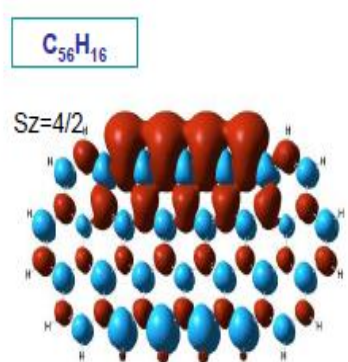

\section{$\mathrm{C}_{64} \mathrm{H}_{19}$}
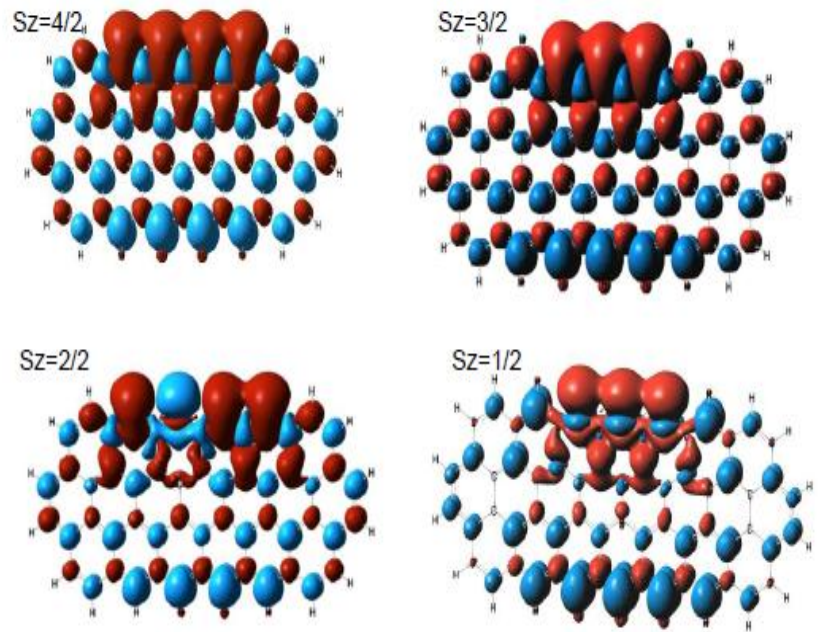

Fig. 3 Spin density map of $\mathrm{Sz}=2 / 2$ and $4 / 2$ in $\mathrm{C}_{56} \mathrm{H}_{16}$, and $\mathrm{Sz}_{\mathrm{z}}=1 / 2$ and $3 / 2$ in $\mathrm{C}_{64} \mathrm{H}_{19}$, where up spins are indicated in red (dark gray), and down spins in blue (light gray) at $0.001 \mathrm{e} / \mathrm{A}^{3}$ contour surface.

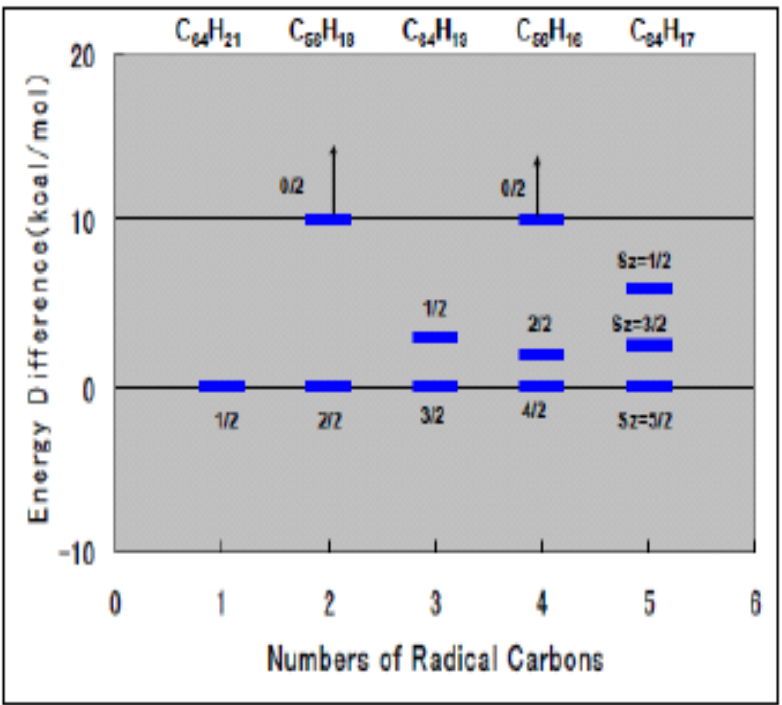

Fig. 4 Energy difference between spin states for radical carbon edge molecules. The highest spin state is the most stable in all molecules. 


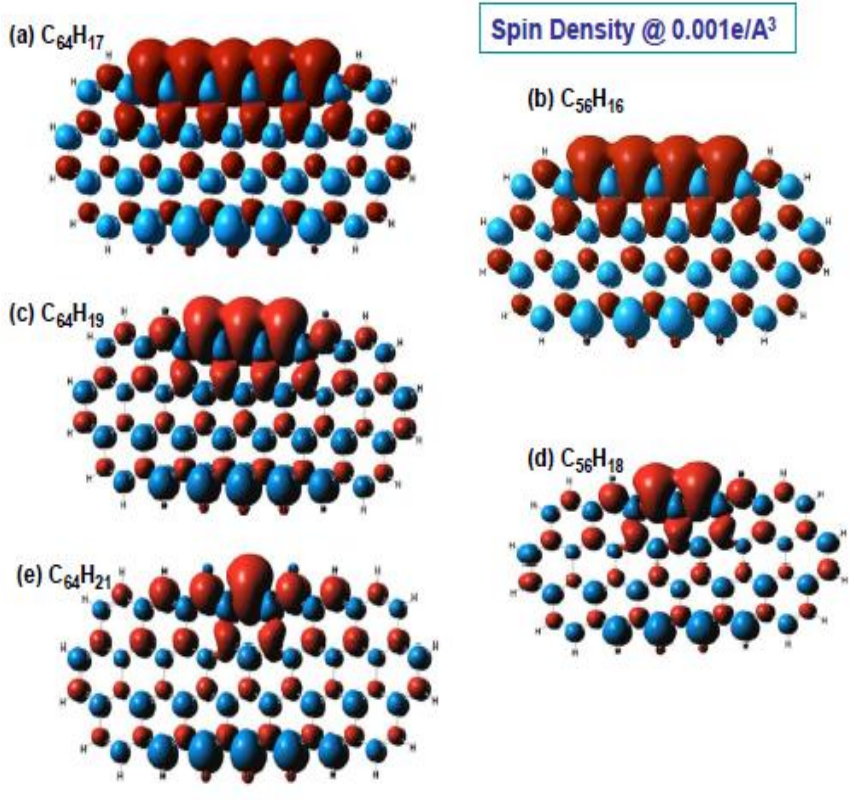

Fig. 5 Spin density map of highest spin states for five molecules. All the edge radical carbon indicates large up-spin density in all molecules. The edge specified spin configuration governs total molecular spin arrangement.

\section{Appling Magnetic Counting Rule to Radical Carbon}

Lieb's rule ${ }^{29)}$ is well-known for magnetic counting of polycyclic aromatic hydrocarbon (PAH). One example is given in Fig. 6. Carbon is classified into two groups: A sites with up-spin $(\mathrm{Sz}=+1 / 2)$, and $\mathrm{B}$ sites with down-spin $(\mathrm{Sz}=-1 / 2)$. These $\mathrm{A}$ and $\mathrm{B}$ sites are alternately aligned one by one. A simple model such as $\mathrm{C}_{14} \mathrm{H}_{10}$ has seven A sites and seven $\mathrm{B}$ sites. The total spin cancels each other out to become $\mathrm{Sz}=0$, which indicates the diamagnetic nature of conventional PAH.

Maruyama et al. ${ }^{30}$ also proposed a rule for dihydrogenated zigzag edge graphene.

Here, we applied a simple magnetic counting rule to the radical carbon edge. As depicted in Fig. 7, we can assume two orbits (triangular marks) with an $\mathrm{sp}^{3}$ like tetrahedral configuration. If two identical electrons occupy those orbits, an up-up parallel spin pair appears due to Hund's rule ${ }^{31), 32}$. This leads to a simple rule where one radical carbon site should be assigned to be $2 \mathrm{~A}(\mathrm{Sz}=2 / 2)$. We applied this simple magnetic counting rule to a molecule, $\mathrm{C}_{64} \mathrm{H}_{17}$, with five radical carbons. As seen in Fig. 8, there are five 2A, twenty-seven $\mathrm{A}$, and thirty-two B sites. The total molecular $\mathrm{Sz}$ is a simple sum of $\mathrm{A}$ and $\mathrm{B}$ numbers, resulting in $5 \mathrm{~A}(\mathrm{Sz}=5 / 2)$. We also applied this rule to other molecules. The $\mathrm{Sz}$ values using this counting rule are summarized in the rightmost column of Table 1 . All the counted Sz values agree with the most stable DFT calculated spin state.

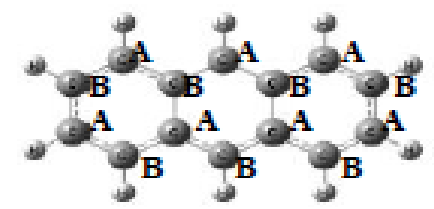

$$
\begin{array}{lc}
\mathrm{A} \times 7 \rightarrow \mathrm{Sz}=+7 / 2 \\
\mathrm{~B} \times 7 \rightarrow & -7 / 2
\end{array}
$$

$$
\mathrm{Sz}=(7 \mathrm{~A}-7 \mathrm{~B}) / 2=0 / 2
$$

Fig.6 Example of magnetic counting rule for polycyclic aromatic hydrocarbon (PAH).

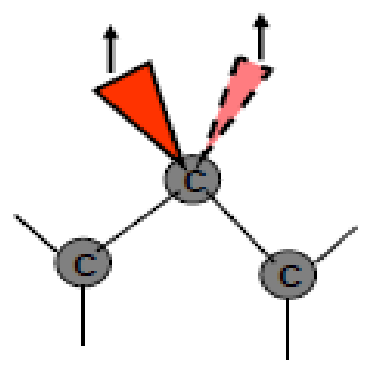

Fig.7 Schematic of molecular orbital image of radical carbon edge with tetrahedral $\mathrm{sp}^{3}$ configuration. Based on Hund's rule, two electrons occupy two $\mathrm{sp}^{3}$ orbits as up-up spin pair.

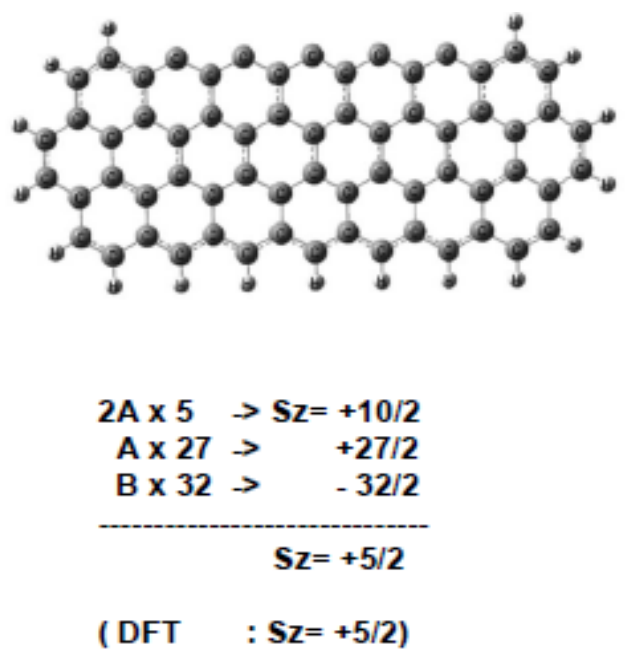

Fig. 8 Total molecular spin $\mathrm{Sz}$ of $\mathrm{C}_{64} \mathrm{H}_{17}$ applying simple magnetic counting rule. A radical carbon edge is assigned to be $2 \mathrm{~A}$. Total molecular spin is the sum of $A$ and $B$ numbers resulting in $\mathrm{Sz}=+5 / 2$, which is consistent with DFT calculations. 


\section{Oxygen Substituted Zigzag Edge}

The tetrahedral configuration of zigzag edge site electrons leads to the question of what happens when there are four electrons at the zigzag edge site. A center atom with four electrons is achieved by oxygen substitution. A simple answer can be estimated from the illustration in Fig. 9. Edge oxygen has $\mathrm{sp}^{3}$ like tetrahedral molecular orbits. Four electrons will occupy the two vacant orbits indicated by the triangles. Two electrons occupy one orbit as an up-down spin pair based on the Pauli principle. Also, two additional electrons occupy another identical orbit as an up-down pair. The total spin cancels each other out, which brings almost no local spin cloud around the oxygen edge. The lower figure in Fig. 9 is a typical model molecule of $\mathrm{C}_{59} \mathrm{O}_{5} \mathrm{H}_{17}$ with five oxygen edges.

The simple speculation above was checked with DFT calculations. The spin density of $\mathrm{C}_{59} \mathrm{O}_{5} \mathrm{H}_{17}$ for $\mathrm{Sz}=5 / 2$, $3 / 2$, and $1 / 2$ are illustrated in Fig. 10. There are many complex up-up spin pairs for $\mathrm{Sz}=5 / 2$ and $3 / 2$, which causes an unfavorable energy increase. The detailed energy difference was calculated and is shown in Fig. 11. For $\mathrm{C}_{59} \mathrm{O}_{5} \mathrm{H}_{17}$, the most stable spin state is $\mathrm{Sz}=1 / 2$, the next most stable is $3 / 2$, and the least stable is $5 / 2$, which coincides with the spin map based speculation above. Changing the oxygen numbers at the zigzag edges from five to one like that in $\mathrm{C}_{59} \mathrm{O}_{5} \mathrm{H}_{17}, \mathrm{C}_{52} \mathrm{O}_{4} \mathrm{H}_{16}$, $\mathrm{C}_{61} \mathrm{O}_{3} \mathrm{H}_{19}, \mathrm{C}_{54} \mathrm{O}_{2} \mathrm{H}_{18}$, and $\mathrm{C}_{63} \mathrm{O}_{1} \mathrm{H}_{21}$, we also found that the lowest spin state was the most stable state in all molecules. We concluded that we could not expect strong magnetism by using oxygen atom substitution.
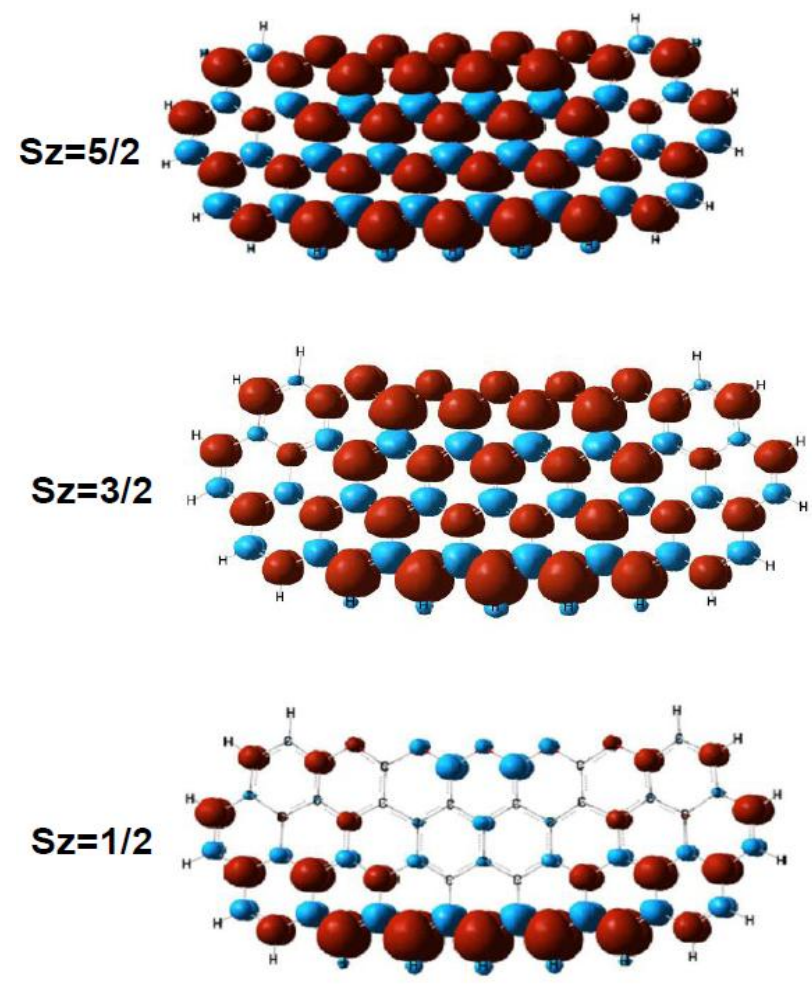

Fig. 10 Spin density map of $\mathrm{C}_{59} \mathrm{O}_{5} \mathrm{H}_{17}$ molecule for $\mathrm{Sz}=5 / 2,3 / 2$, and $1 / 2$. There are serious up-up spin pairs for the higher spin state, which causes an unfavorable energy increase. The lowest spin state of $\mathrm{Sz}=1 / 2$ is energetically favorable and stable.

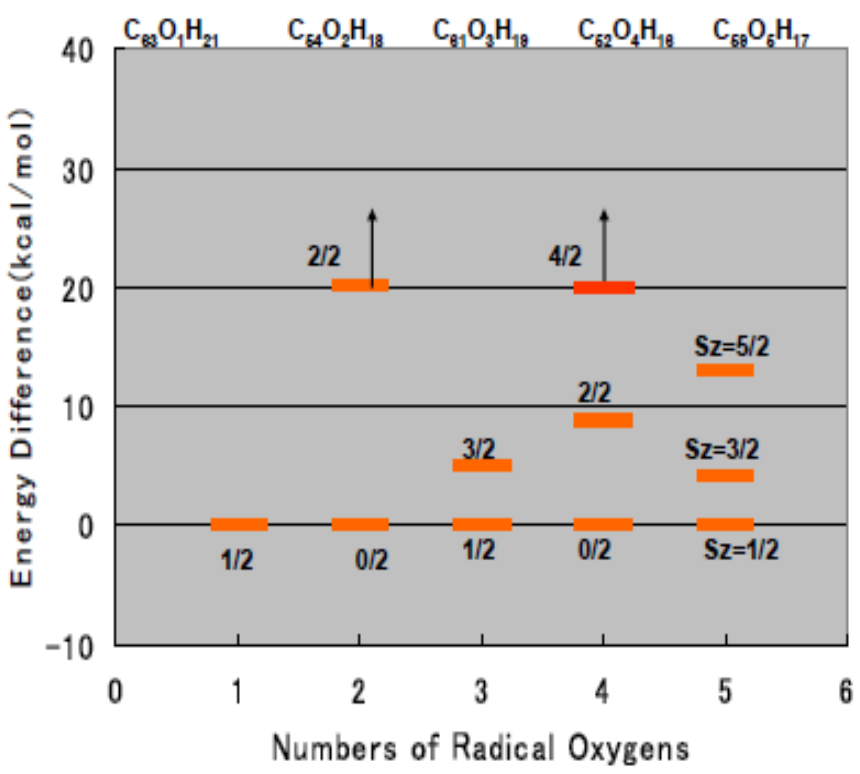

Fig.11 Comparison of molecular energy for five molecules with oxygen-substituted zigzag edges. The lowest spin state is most stable in all molecules. 


\section{Conclusion}

Recent experiments have indicated the capability of $\mathrm{RT}$ ferromagnetism in graphite-like materials. We modeled a radical carbon edge graphene-like molecule to attempt a basic understanding. We analyzed multiple spin states based on first principles density function theory. Molecules with radical carbon zigzag edges such as $\mathrm{C}_{64} \mathrm{H}_{17}$ (five radical carbons), $\mathrm{C}_{56} \mathrm{H}_{16}$ (four), $\mathrm{C}_{64} \mathrm{H}_{19}$ (three), $\mathrm{C}_{56} \mathrm{H}_{18}$ (two), and $\mathrm{C}_{64} \mathrm{H}_{21}$ (one) demonstrated that the highest possible spin state was the most stable in all molecules. Spin density at the radical carbon edge had a large up-spin cloud, which originated from two up-up spins due to Hund's rule. A simple magnetic counting rule was applied to give $\mathrm{Sz}=+2 / 2$ to one radical carbon site, $\mathrm{Sz}=-1 / 2$ to the nearest carbon, and $+1 / 2$ to the second nearest one. Total molecular $\mathrm{Sz}$ was the sum of those numbers. By applying this counting rule to the five molecules, we were able to reproduce all the stable spin states, which agreed with the DFT results. In addition, we also applied a similar idea to a case with four electrons, which meant oxygen atom substitution at the zigzag edge. Two tetrahedral orbits were occupied by four spins that cancelled each other out and demonstrated weak molecular magnetism. This was confirmed through detailed DFT calculations done on five molecules with different numbers of oxygen atoms. In conclusion, we can expect strong magnetism in radical carbon zigzag edge graphene-like molecule.

\section{Acknowledgements}

Narjes Gorjizadeh would like to thank the crew of the Center for Computational Materials Science in the Institute for Materials Research at Tohoku University, the Global COE Program of "Materials Integration, Tohoku University," and MEXT, Japan, for its financial support.

\section{References}

1) A. A. Ovchinnikov and V. N. Spector:

Synth. Met., 27, B615 (1988)

2) K. Murata, H. Ueda, and K. Kawaguchi:

Synth. Met., 44, 357 (1991).

3) K. Murata, H. Ushijima, H. Ueda, and K. Kawaguchi: J.

Chem. Soc. Chem. Commun., 7,567 (1992).

4) J. Miller: Inorg. Chem., 39, 4392 (2000).

5) Y. Shiroishi, F. Fukuda, I. Tagawa, H. Iwasaki, S.

Takenoiri, H. Tanaka, M. Mutoh, and N. Yoshikawa: IEEE

Trans. on Magnetics, 45, 3816 (2009).

6) Y. Shiroishi: Magune, 5, (2010), Review in Japanese.

7) M. Ohishi, M. Shiraishi, R. Nouchi, T. Nozaki, T. Shinjyo, and Y. Suzuki: Jpn. J. Appl. Phys. 46, L605 (2007).

8) S. Shiraishi: Surface Science、29、310 (2008) , Review in Japanese.

9) N. Tombros, C. Jozsa, M. Popinciuc, H. T. Jonkman, and B. J. van Wees: Nature, 448, 571 (2007).
10) P. Esquinazi, D. Spemann, R. Hohne, A. Setzer, K. Han, and T. Butz: Phys. Rev. Lett., 91, 227201 (2003)

11) K. Kamishima, T. Noda, F. Kadonome, K. Kakizaki, and N. Hiratsuka: J.of Magnetism and Magnetic Materials 310, e346 (2007)

12) T. Saito, D. Nishio-Hamane, S. Yoshii, and T. Nojima: Appl. Phys. Letters, 98, 052506 (2011).

13) Y. Wang, Y. Huang, Y. Song, X. Zhang, Y. Ma, J. Liang, and Y. Chen: Nano Letters , 9, 220 (2009).

14) J. Cervenka, M. Katsnelson, and C. Flips: Nature Physics online Oct. 04 (2009).

15) H. Ohldag, P. Esquinazi, E. Arenholz, D. Spemann, M.

Rothermal, A. Setzer, and T. Butz: New Journal of Physics, 12, 123012 (2010).

16) M. Fujita, K. Wakabayashi, K. Nakada, and K. Kusakabe: J. Phys. Soc. Jpn . 65, 1920 (1996).

17) K. Nakada, M. Fujita, G. Dresselhaus, and M.

Dresselhaus: Phy. Rev. B 54, 17954 (1996).

18) K. Wakabayashi, M. Fujita, H. Ajiki, and M. Sigrist: Phys. Rev. B 59, 8271 (1999).

19) Y. Miyamoto, K. Nakada, and M. Fujita: Phys. Rev. B, 59, 9858 (1999).

20) J. Berashevich and T. Chakraborty: Phys. Rev. B, 80, 115430 (2009).

21) H. Zheng and W. Duley: Phys. Rev. B, 78, 045421 (2008) 22) D. E. Jiang, B. G. Sumpter, and S. Dai: J. Chem. Phys., 127,124703 (2007)

23) X. Gao, Z. Zhuo, Y. Zhao, S. Nagase, S. B. Zhang, and Z. Chen: J. Phys. Chem. C112, 12677 (2008).

24) T. Wassman, A. P. Seitsonen, A. M. Saitta, M. Lazzeri, and F. Mauri: Phys. Rev. Lett., 101, 096402(2008).

25) K. Kusakabe and M. Maruyama: Phys. Rev. B, 67, 092406 (2003).

26) M. Maruyama and K. Kusakabe: J. Phys. Soc. Jpn, .73, 656 (2004).

27) N. Ota, N. Gorjizadeh, and Y. Kawazoe: J. Magn. Soc.

Japan 34, 573 (2010).

28) N.Ota, N.Gorjizadeh and Y.Kawazoe:

J. Magn. Soc. Japan 35, 360 (2011).

29) E. H. Lieb: Phys. Rev. Lett. 62,1201 (1989).

30) M. Maruyama, K. Kusakabe, S. Tsuneyuki, K. Akagi, Y.

Yoshimoto, and J. Yamauchi: J. of Physics and Chemistry of Solids 65,119 (2004).

31) F. Hund: Z Physik 33, 345 (1925).

32) Y. Maruyama, K. Hongo, M. Tachikawa, Y. Kawazoe, and

H. Yasuhara: Int. J. Quant. Chem., 108, 731 (2008).

33) P. Hohenberg and W. Kohn: Phys. Rev., 136 , B864 (1964).

34) W. Kohn and L. Sham: Phys. Rev., 140, A1133 (1965).

35) J. P. Perdew, K. Burk, and M. Ernzerhof: Phy. Rev. Lett., 77, 3865 (1966).

36) R. Ditchfield, W. Hehre, and J. Pople: J. Chem. Phys. 54, 724 (1971).

Received April 12, 2011; Accepted July 13, 2011 